\title{
Billion-Fold Enhancement in Sensitivity of Nuclear Magnetic Resonance Spectroscopy for Magnesium Ions in Solution
}

\author{
Alexander Gottberg, ${ }^{[a]}$ Monika Stachura, ${ }^{*[a, b]}$ Magdalena Kowalska, ${ }^{[a]}$ Mark L. Bissell, ${ }^{[c]}$ \\ Vaida Arcisauskaite, ${ }^{[b]}$ Klaus Blaum, ${ }^{[\mathrm{d}]}$ Alexander Helmke, ${ }^{[\mathrm{e}]}$ Karl Johnston, ${ }^{[\mathrm{f}]}$ Kim Kreim, ${ }^{[\mathrm{d}]}$ \\ Flemming H. Larsen, ${ }^{[\mathrm{g}]}$ Rainer Neugart, ${ }^{[\mathrm{h}]}$ Gerda Neyens, ${ }^{[\mathrm{c}]}$ Ronald F. Garcia Ruiz, ${ }^{[\mathrm{c}]}$ \\ Daniel Szunyogh, ${ }^{[]]}$Peter W. Thulstrup,${ }^{[b]}$ Deyan T. Yordanov, ${ }^{[d]}$ and Lars Hemmingsen ${ }^{[b]}$
}

$\beta$-nuclear magnetic resonance (NMR) spectroscopy is highly sensitive compared to conventional NMR spectroscopy, and may be applied for several elements across the periodic table. $\beta$-NMR has previously been successfully applied in the fields of nuclear and solid-state physics. In this work, $\beta$-NMR is applied, for the first time, to record an NMR spectrum for a species in solution. ${ }^{31} \mathrm{Mg} \beta$-NMR spectra are measured for as few as $10^{7}$ magnesium ions in ionic liquid (EMIM-AC) within minutes, as a prototypical test case. Resonances are observed at 3882.9 and $3887.2 \mathrm{kHz}$ in an external field of $0.3 \mathrm{~T}$. The key achievement of the current work is to demonstrate that $\beta$-NMR is applicable for the analysis of species in solution, and thus represents a novel spectroscopic technique for use in general chemistry and potentially in biochemistry.

Nuclear magnetic resonance (NMR) spectroscopy is currently the most versatile spectroscopic technique for the characterization of molecular structure and dynamics in solution. However, the main limitation of NMR is sensitivity, as $>0.1 \mathrm{~mm}$ concentrations (or $>10^{16}$ of probe nuclei) are typically required. In addition, NMR spectroscopy depends on the availability of isotopes of a given element with appropriate nuclear properties, and is most straightforwardly applied to nuclei with spin $=1 / 2$, that is, not all elements may be interrogated. To address the issue of sensitivity, dynamic nuclear polarization (DNP) has been utilized, enhancing the sensitivity substantially by transfer of polarization from unpaired electrons in an added radical. ${ }^{[1,2]}$ Similarly, NMR techniques that allow for the observation of as few as hundreds of nuclear spins in volumes as small as a few cubic nanometers have recently been developed, ${ }^{[3,4]}$ and even a single proton in a nuclear physics experiment, providing a high precision value of the proton magnetic moment. ${ }^{[5]}$ In this work, we present the first application of a complementary NMR technique, $\beta$-NMR spectroscopy, ${ }_{1}^{[6]}$ to an ionic-liquid solution containing $\mathrm{Mg}^{2+}$ ions. The particular advantages of $\beta$ NMR are extreme sensitivity and access to elements that are difficult to detect in solution by using conventional NMR, such as $\mathrm{Mg}$ and potentially $\mathrm{Cu}$.

The two most prominent differences between conventional NMR and $\beta$-NMR spectroscopy are 1) that the nuclei are polarized prior to implantation into the sample in $\beta$-NMR and 2 ) the manner in which resonances are detected. $\beta$-NMR relies on the detection of $\beta$-particles from the decay of radioactive nuclei, and thus benefits from the high sensitivity of radiotracer experiments. Initially, an ion beam of spin-polarized nuclei is implanted into the material of interest. As the direction of emission of the $\beta$-particles depends on the nuclear spin direction, the $\beta$-particles are emitted anisotropically. ${ }^{[7]}$ In analogy to conventional NMR spectroscopy, the probe nuclei in the system of interest are placed in an external magnetic field and exposed [a] Dr. A. Gottberg, ${ }^{+}$Dr. M. Stachura, ${ }^{+}$Dr. M. Kowalska

CERN, 1211 Genevé 23 (Switzerland)

Fax: $(+41) 227678990$

E-mail:monika.stachura@cern.ch

[b] Dr. M. Stachura, Dr. V. Arcisauskaite, Prof. P. W. Thulstrup,

Prof. L. Hemmingsen

Kemisk Institut, Københavns Universitet

Universitetsparken 5, 2000 København (Denmark)

E-mail:Ihe@chem.ku.dk

[c] Dr. M. L. Bissell, Prof. G. Neyens, R. F. G. Ruiz Instituut voor Kern- en Stralingsfysica, KU Leuven Celestijnenlaan 200 D, 3001 Leuven (Belgium)

[d] Prof. K. Blaum, Dr. K. Kreim, Dr. D. T. Yordanov Max-Planck-Institut für Kernphysik

Saupfercheckweg 1, 69117 Heidelberg (Germany)

[e] A. Helmke

Humedics $\mathrm{GmbH}$

Marie-Elisabeth-Lüders-Str. 1, 10625 Berlin (Germany)

\section{[f] Dr. K. Johnston}

Universität des Saarlandes, Experimentalphysik

66123 Saarbrucken (Germany)

[g] Prof. F. H. Larsen

Institut for Fødevarevidenskab, Københavns Universitet Rolighedsvej 30, 1958 Frederiksberg C (Denmark)

[h] Prof. R. Neugart

Institut für Kernchemie, Universität Mainz

Fritz-Straßmann-Weg 2, 55128 Mainz (Germany)

[i] D. Szunyogh

MTA-SZTE Bioszervetlen Kémiai Kutatócsoport

Dóm tér 7, 6720 Szeged (Hungary)

$\left.{ }^{[+}\right]$These authors contributed equally to this work.

Supporting Information for this article is available on the WWW under http://dx.doi.org/10.1002/cphc.201402619. 
to radio-frequency electromagnetic radiation. At the resonance frequency, transitions between magnetic sub-states occur, giving rise to a loss of nuclear spin polarization and, thus, loss of anisotropy in the $\beta$-particle emission. Over recent decades, $\beta$-NMR spectroscopy has successfully been applied under highvacuum conditions in nuclear physics to measure nuclear spins, magnetic dipole moments, and electric quadrupole moments, ${ }^{[8-11]}$ as well as in solid-state physics for studying radiation damage, ${ }^{[12]}$ location of impurities in semiconductors, ${ }^{[13,14]}$ and the diffusion and magnetic properties of thin films. ${ }^{[15,16]}$

The properties recorded in solid-state applications are essentially the same as those of interest in solution, that is, chemical shifts or relaxation times. $\beta$-NMR experiments on non-crystalline materials have previously been used for samples such as liquid alloys, ${ }^{[12]}$ liquid neon, ${ }^{[17]}$ glasses, and the glass transition of hydrated $\mathrm{LiCl}^{[18]}$ but no NMR spectrum has been recorded for species in solution. This is presumably because of technical obstacles, most notably 1) the challenge of having a liquid sample/vacuum interface, 2) possible beam attenuation or loss of spin polarization through collisions with gas-phase molecules that are present above the sample, dictated by the vapor pressure of the liquid, or in the liquid, and 3) whether the spinpolarized ions will bind fast enough (within the lifetime of the radionuclide) to the relevant binding site in solution at low concentrations.

In this work, we present the application of ${ }^{31} \mathrm{Mg}\left(T_{1 / 2}=\right.$ $230 \mathrm{~ms}, I=1 / 2)^{[9,10]} \beta$-NMR spectroscopy to magnesium ions in ionic-liquid solution as a proof-of-principle, using a novel prototype on-line setup specifically designed for liquid samples at the CERN-ISOLDE facility. The results indicate that even aqueous solutions can be explored with $\beta$-NMR, broadening the scope from chemistal to biochemical applications.

Ionic liquid 1-ethyl-3-methyl-imidazolium acetate (EMIM-AC) was selected as the solvent, primarily because of its exceedingly low vapor pressure. In addition, with acetate as the anion, it is expected to form oxygen-rich first-coordination spheres for the magnesium ions. Additionally, the use of ionic liquids as alternative solvents to water has advanced significantly over the past two decades, ${ }^{[19]}$ that is, the properties of these solvents for example for organic synthesis and the coordination chemistry of metal ions, often used as catalysts, are of increasing and general interest. ${ }^{31} \mathrm{Mg}$ was selected as radioisotope for the $\beta$ NMR experiments because spin-polarized ${ }^{31} \mathrm{Mg}^{+}$beams can be produced at sufficient yield, ${ }^{[10]}$ the nuclear decay is suitable, and ${ }^{31} \mathrm{Mg}$ has $I=1 / 2$ and will, therefore, only display magnetic interactions. In contrast, conventional NMR on magnesium is complex, because the only stable spin-bearing isotope, ${ }^{25} \mathrm{Mg}$, has a modest magnetic moment and is a quadrupolar nuclide $(I=5 / 2)$. Furthermore, $\mathrm{Mg}^{2+}$ is spectroscopically silent in most standard spectroscopic techniques, as it is a closed-shell ion. Finally, $\mathrm{Mg}^{2+}$ is essential in biochemistry, where it is involved in phosphate chemistry and in photosynthesis as an integral component of chlorophyll. ${ }^{[20]}$ Thus, a novel technique could significantly advance the elucidation of magnesium coordination chemistry and biochemistry.

As the ionic liquid has a very low vapor pressure it was possible to systematically investigate the beam transmission from

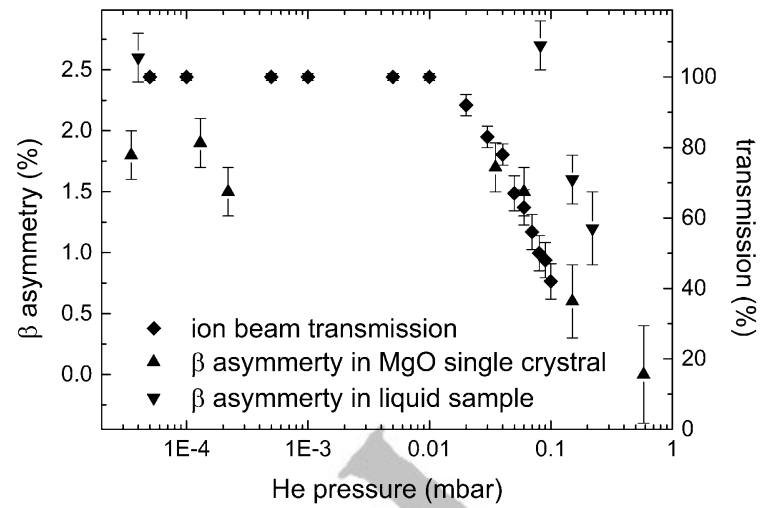

Figure 1. Right) ${ }^{39} \mathrm{~K}^{+}$beam transmission through $\mathrm{He}$ gas at varying pressures $(\diamond)$. Left) $\beta$-asymmetry of ${ }^{31} \mathrm{Mg}^{+}$implanted into a $\mathrm{MgO}$ single crystal $(\boldsymbol{\Delta})$ or into ionic liquid EMIM-AC $(\boldsymbol{\nabla})$.

the production site to the sample by introducing He gas in the sample chamber to create different levels of pressure, see Figure 1. $\square$ ok? $\square$

The transmission of a $50 \mathrm{keV}$ beam of ${ }^{39} \mathrm{~K}^{+}$through the He gas was recorded. ${ }^{39} \mathrm{~K}^{+}$was used to probe the transmission, because the radioactive ${ }^{31} \mathrm{Mg}^{+}$beam time is very precious and ${ }^{39} \mathrm{~K}$ is a stable isotope with comparable mass. Clearly, the beam transmission decreases significantly above pressures of approximately $0.1 \mathrm{mbar}$. With improvements to the experimental design, it should be possible to advance into a range (ca. mbar) where water may be used as the solvent, which is the ultimate goal for applications in both chemistry and biochemistry. By simple geometric arguments, we estimate that a minor fraction (ca. 20\%) of the beam was deposited in the aluminum pipe, constituting the last part of a differential pumping system, see Figure $2 \mathrm{~B}$. This is corroborated by the fact that $\beta$-particles from ${ }^{31} \mathrm{Mg}$ were recorded even when no sample was present in the chamber, that is, under conditions where the beam should just pass through, potentially giving rise to a $\beta$-NMR resonance from ${ }^{31} \mathrm{Mg}$ deposited in the aluminum, vide infra. Beam transmission in itself, however, is only a necessary prerequisite for $\beta$-NMR experiments, where the nuclear spin polarization must also be preserved. This was probed by using a $61 \mathrm{keV}$ beam of ${ }^{31} \mathrm{Mg}^{+}$implanted into a $\mathrm{MgO}$ single crystal as well as into the ionic liquid, and the $\beta$ asymmetry was recorded as a function of the He gas pressure in the chamber, see Figure 1. In both cases, the asymmetry decreases in the same pressure range as the beam transmission, indicating that spin polarization is indeed preserved to a significant extent during the passage of He gas. A priori, it cannot be excluded that spin polarization is lost during implantation into the liquid, although this is not the case in the solid state. ${ }^{[11]}$ The data in Figure 1 demonstrate that spin polarization persists in the liquid, long enough for the $\beta$-asymmetry to be recorded. The data display an additional interesting feature, namely the fact that the observed asymmetry in the ionic liquid is even larger than in the MgO crystal. This implies that the spin-lattice relaxation time, $T_{1}$, is longer in the ionic liquid than in the $\mathrm{MgO}$ crystal. An attempt to determine $T_{1}$ in the ionic liquid revealed that it was longer than the lifetime of the 

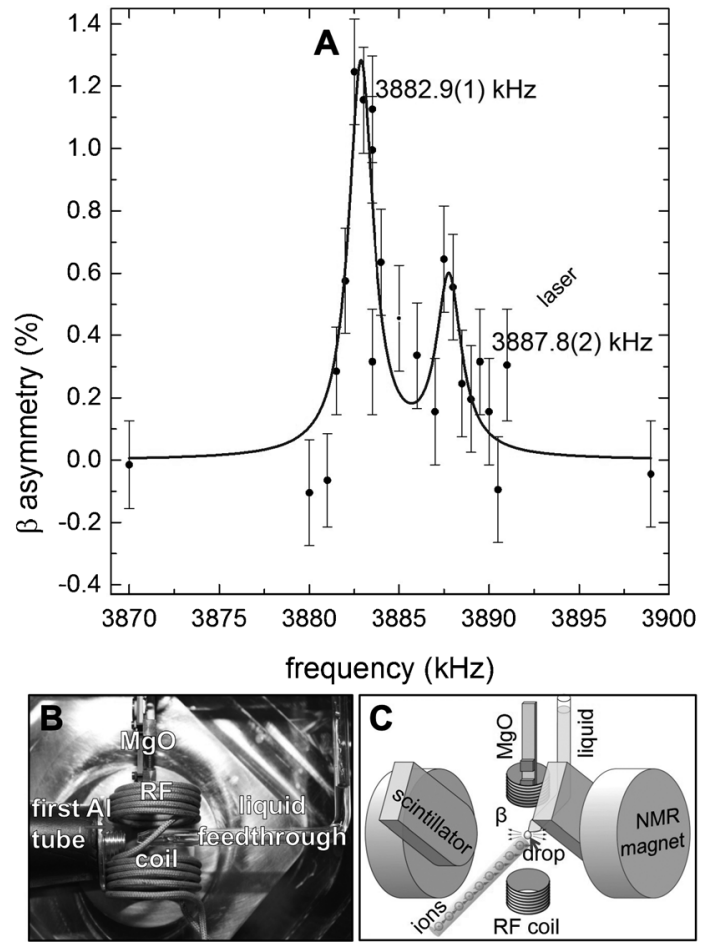

Figure 2. A) $\beta$-NMR spectrum of ${ }^{31} \mathrm{Mg}^{+}$implanted into ionic liquid EMIM-AC in an external magnetic field of $0.3 \mathrm{~T}$. Experimental data points are shown with error bars, and the fit is displayed as a full line. B) The core of the experimental setup, showing the aluminum pipe (part of a differential pumping system), the RF coils, the system for liquid feed-through, and the MgO single crystal. C) Simplified sketch of the experimental setup, including the incoming nuclear spin-polarized ${ }^{31} \mathrm{Mg}^{+}$ion beam, the $\beta$-particle scintillation detectors, and the NMR magnet.

radioisotope (230 ms), that is, ${ }^{31} \mathrm{Mg}$ decayed before spin polarization was lost.

The beam of monovalent ${ }^{31} \mathrm{Mg}^{+}$is assumed to be rapidly oxidized to the stable oxidation state, $\mathrm{Mg}^{2+}$, once it is implanted into the ionic liquid solution. The ${ }^{31} \mathrm{Mg}^{+}$beam has an energy of $61 \mathrm{keV}$, which is low from a nuclear physics perspective, but high from a chemical viewpoint. The implantation of these high-energy ${ }^{31} \mathrm{Mg}^{+}$radicals may give rise to the formation of transient radical species, potentially leading to rapid depolarization of the nuclear spin, and thus a lost fraction of signal in the $\beta$-NMR experiment. However, it is assumed that such reactions occur on a time scale much shorter than the lifetime of ${ }^{31} \mathrm{Mg}$ and, therefore, that any remaining signals after implantation reflect the coordination chemistry of $\mathrm{Mg}^{2+}$.

In Figure $2 \mathrm{~A}$, the $\beta$-NMR spectrum of ${ }^{31} \mathrm{Mg}^{+}$implanted into the ionic liquid is displayed, illustrating that two resonances are observed. Moreover, the $\beta$-asymmetry increases significantly upon the introduction of the ionic liquid into the sample chamber, see Figure 1 and S1, demonstrating that at least part of the NMR signal arises from ${ }^{31} \mathrm{Mg}$ implanted into the ionic liquid. These observations provide the qualitative proof-of-principle that $\beta$-NMR spectroscopy may be applied to species in solution. The two observed resonances imply that the magnesium nuclei experience two different binding sites with different shielding. The spectrum was recorded twice, and it was reproducible. The difference in resonance frequency between the two signals corresponds to a chemical shift of approximately $1300 \mathrm{ppm}$, which is far beyond the usual range of chemical shifts of about $200 \mathrm{ppm}$ observed for $\mathrm{Mg}^{2+}$-containing compounds when using conventional ${ }^{25} \mathrm{Mg}$ NMR spectroscopy. ${ }^{[21]}$ However, it is well established that the Knight shift, owing to polarization of conduction electrons in metals, is typically of the order of $1000 \mathrm{ppm}$; it is also reported to range from 990 to $1750 \mathrm{ppm}$, specifically for ${ }^{25} \mathrm{Mg}$ in $\mathrm{Mg}$ metal as well as in alloys of $\mathrm{Mg}$ and Al. ${ }^{[22]}$ This correlates well with the discussion concerning the loss of beam in the aluminum pipe, vide supra, indicating that a fraction of ${ }^{31} \mathrm{Mg}^{+}$is deposited in the aluminum. The Knight shift gives rise to an up-field shift, corresponding to a higher resonance frequency, and thus represents the resonance at $3887.8(2) \mathrm{kHz}$. This may be considered to be an artifact of the current measurements, but could also be exploited as an internal chemical-shift reference, which gives a resonance conveniently far from the range where chemically and biochemically interesting resonances are observed; therefore, no serious overlap of signals will occur. The other resonance is then found in the same range as that observed for $\mathrm{Mg}^{2+}$-containing compounds in conventional NMR spectroscopy, ${ }^{[21]}$ that is, within approximately $100 \mathrm{ppm}$ of an $11 \mathrm{M} \mathrm{MgCl} 2$ reference, presumably reflecting the coordination of the carboxylates in the ionic-liquid anion.

To complement the analysis of the $\beta$-NMR experiments, conventional ${ }^{25} \mathrm{Mg}$ NMR experiments were conducted on a similar sample, although with a much higher $\mathrm{Mg}^{2+}$ concentration, see Figure S3. A single resonance was observed at $10 \mathrm{ppm}$ with respect to $11 \mathrm{M} \mathrm{MgCl}$. Attempts to detect other resonances within \pm 1500 ppm were carried out, but none were observed, supporting the above interpretation of the $\beta$-NMR resonances.

In summary, we present the first application of $\beta$-NMR to record an NMR spectrum of a species in solution. The main advantage of this technique is the extreme sensitivity and, from a general chemistry perspective, the fact that several isotopes that can be spin-polarized are accessible at radioactive ionbeam facilities worldwide. A limiting factor is that the spin-polarized ions are required to bind to the binding site of interest within the lifetime of the radioisotope, that is, typically within 0.1-1 s after implantation into the liquid. Thus, an obvious applications lie in the coordination chemistry and biochemistry of metal ions, which display modest-to-fast ligand-exchange reactions, and this is indeed the case for most biologically relevant metal ions. From a biochemical perspective, the interaction of essential metal ions, which are spectroscopically silent in most techniques, such as $\mathrm{Na}^{+}, \mathrm{Mg}^{2+}, \mathrm{K}^{+}, \mathrm{Ca}^{2+}, \mathrm{Cu}^{+}$, and $\mathrm{Zn}^{2+}$, with biological macromolecules at physiologically relevant concentrations, may be explored, opening new avenues of research in the fields of metal-ion transport, nucleic acids, as well as redox-active and catalytic metalloproteins.

\section{Experimental Section}

\section{及-NMR Spectroscopy}

The sample used for $\beta$-NMR spectroscopy was prepared by dissolving $\mathrm{Mg}(\mathrm{Ac})_{2} \cdot 4 \mathrm{H}_{2} \mathrm{O}$ (32.17 mg; purchased from Sigma-Aldrich; $99 \%$ 
purity, $\left.M=214.46 \mathrm{~g} \mathrm{~mol}^{-1}\right)$ in 1-ethyl-3-methyl-imidazolium acetate (7.5 mL; purchased from Sigma-Aldrich; $97 \%$ purity), leading to a final concentration of $\mathrm{Mg}^{2+}$ of $20 \mathrm{~mm}$. In experiments where only trace amounts of radioactive isotopes are used, even a small amount of high-affinity binding sites at surfaces, for example, can affect the results. This is not expected to occur for $\mathrm{Mg}^{2+}$, but, to eliminate this potential systematic error, we added non-radioactive magnesium. The sample was placed under vacuum $2 \mathrm{~h}$ prior to the $\beta$-NMR experiment to avoid changes during the experiment. During transfer of the sample to the beam line, it was exposed to air for some minutes.

The beam of ${ }^{31} \mathrm{Mg}^{+}$nuclei were produced at ISOLDE at CERN, and polarized "on-line" through interactions with a collinear circularly polarized laser beam. The laser-induced electron polarization was transferred to the nuclei through the hyperfine interaction, leading to $20-50 \%$ nuclear polarization. The polarized beam was implanted in the liquid host after passing through a differential pumping section. The experimental setup applied in this work was that of the COLLAPS collaboration at ISOLDE-CERN ${ }^{[10]}$ equipped with a novel differential pumping system and a sample chamber that are described elsewhere. $\mathbf{D}$ Reference required $\mathbf{D}$ The data in Figure 2 were fitted with two Gaussian peak profiles. The common width and the individual peak intensities and positions were left open in the fit. At ISOLDE-CERN, $10^{4}$ optically polarized ${ }^{31} \mathrm{Mg}^{+}$ions were implanted per second into a solution of $20 \mathrm{~mm} \mathrm{Mg}(\mathrm{Ac})_{2}$ in 1ethyl-3-methyl-imidazolium acetate (EMIM-AC).

\section{NMR Spectroscopy}

The sample used for conventional ${ }^{25} \mathrm{Mg}$ NMR spectroscopy was prepared by dissolving $\mathrm{Mg}(\mathrm{Ac})_{2} \cdot 4 \mathrm{H}_{2} \mathrm{O}$ (71.79 mg) in 1-ethyl-3methyl-imidazolium acetate $(1.19381 \mathrm{~g}$, corresponding to $1.162 \mathrm{~mL} ; \rho=1.027 \mathrm{~g} \mathrm{~mL}^{-1}$ ), leading to a final concentration of $\mathrm{Mg}^{2+}$ of $288 \mathrm{~mm}$. Liquid-state NMR spectra were recorded on a Bruker Avance DRX-500 (11.7 T) spectrometer, operating at Larmor frequencies of 500.13 and $30.62 \mathrm{MHz}$ for ${ }^{1} \mathrm{H}$ and ${ }^{25} \mathrm{Mg}$, respectively. All spectra were recorded in a double-tuned $\mathrm{BBI}$ probe equipped for $5 \mathrm{~mm}$ (o.d.) sample tubes. The ${ }^{1} \mathrm{H}$ NMR spectra were recorded by a single-pulse experiment, using a recycle delay of $5 \mathrm{~s}$, 8 scans, a spectral width of $50 \mathrm{kHz}$, and an acquisition time of $0.328 \mathrm{~s}$. The ${ }^{25} \mathrm{Mg}$ NMR spectra were recorded by using a spin-echo experiment with a delay of $500 \mu \mathrm{s}, 16384$ scans, a recycle delay of $1 \mathrm{~s}$, a spectral width of $100 \mathrm{kHz}$, and an acquisition time of $0.164 \mathrm{~s}$. Experiments were performed at temperatures of 25, 40, 50,60, and $70^{\circ} \mathrm{C}$. Subsequently spectra were apodized by Lorentzian-line broadenings of 0.3 and $10 \mathrm{~Hz}$ for ${ }^{1} \mathrm{H}$ and ${ }^{25} \mathrm{Mg}$, respectively, prior to Fourier transformation. ${ }^{1} \mathrm{H}$ NMR spectra were referenced to the methyl resonance at $0.824 \mathrm{ppm}$, whereas ${ }^{25} \mathrm{Mg}$ spectra were referenced to an external sample of $11 \mathrm{M} \mathrm{Mg}(\mathrm{Cl})_{2}$ in $\mathrm{D}_{2} \mathrm{O}$.

\section{Acknowledgements}

CERN-ISOLDE is acknowledged for beam-time grant 188 and technical support; the Danish Center for Scientific Computing for resources, the Danish Council for Independent Research | Natural
Sciences and FP7 ENSAR (no. 262010) are thanked for financial support; Dr. Y. Blumenfeld, Dr. H. W. Adolph, and Dr. J. Vibenholt are also acknowledged for fruitful discussions and support.

Keywords: $\beta$-NMR • ionic liquids • magnesium • NMR spectroscopy $\cdot$ ultrasensitivity

[1] R. V. Shchepin, A. M. Coffey, K. W. Waddell, E. Y. Chekmenev, Anal. Chem. 2014, 86, 5601.

[2] J. H. Ardenkjær-Larsen, B. Fridlund, A. Gram, G. Hansson, L. Hansson, M. H. Lerche, R. Servin, M. Thaning, K. Golman, Proc. Natl. Acad. Sci. USA 2003, 100, 10158

[3] T. Staudacher, F. Shi, S. Pezzagna, J. Meijer, J. Du, C. A. Meriles, F. Reinhard, J. Wrachtrup, Science 2013, 339, 561.

[4] H. J. Mamin, M. Kim, M. H. Sherwood, C. T. Rettner, K. Ohno, D. D. Awschalom, D. Rugar, Science 2013, 339, 557.

[5] A. Mooser, S. Ulmer, K. Blaum, K. Franke, H. Kracke, C. Leiteritz, W. Quint, C. C. Rodegheri, C. Smorra, J. Walz, Nature 2014, 509, 596.

[6] a) M. Matthias, B. Olsen, D. A. Shirley, J. E. Templeton, R. M. Steffen, Phys. Rev. A 1971, 4, 1626; b) I. S. Mackenzie, J. Phys. E 1986, 19, 324.

[7] W. D. Brewer, Hyperfine Interact. 1982, 12, 173.

[8] W. Geithner, S. Kappertz, M. Keim, P. Lievens, R. Neugart, L. Vermeeren, S. Wilbert, V. N. Fedoseyev, U. Köster, V. I. Mishin, V. Sebastian, Phys. Rev. Lett. 1999, 83, 3792.

[9] G. Neyens, M. Kowalska, D. Yordanov, K. Blaum, P. Himpe, P. Lievens, S. Mallion, R. Neugart, N. Vermeulen, Y. Utsuno, T. Otsuka, Phys. Rev. Lett. 2005, 94, 022501.

[10] M. Kowalska, D. T. Yordanov, K. Blaum, P. Himpe, P. Lievens, S. Mallion, R. Neugart, G. Neyens, N. Vermeulen, Phys. Rev. C 2008, 77, 34307.

[11] R. Neugart, D. L. Balabanski, K. Blaum, D. Borremans, P. Himpe, P. Lievens, S. Mallion, G. Neyens, N. Vermeulen, D. T. Yordanov, Phys. Rev. Lett. 2008, 101, 132502.

[12] H. Ackermann, P. Heitjans, H. J. Stöckmann, Hyperfine Interact. 1985, 25, 395.

[13] H. Ackermann in Hyperfine Interactions of Defects in Semiconductors, (Ed.: G. Langouche) Elsevier, 1992, pp. 325-337.

[14] A. I. Mansour, G. D. Morris, Z. Salman, d, K. H. Chow, , T. Dunlop, J. Jung, I. Fan, W. A. MacFarlane, R. F. Kiefl, f, g, T. J. Parolin, H. aadaoui, D. Wang, M. D. Hossain, Q. Song, M. Smadella, O. Mosendz, B. Kardasz, B. Heinrich. ,Physica B 2007, 401-402, 662.

[15] I. McKenzie, M. Harada, R. F. Kiefl, C. D. P. Levy, W. A. MacFarlane, G. D. Morris, S.-I. Ogata, M. R. Pearson, J. Sugiyama, J. Am. Chem. Soc. 2014, 136, 7833.

[16] G. D. Morris, W. A. MacFarlane, K. H. Chow, Z. Salman, D. J. Arseneau, S. Daviel, A. Hatakeyama, S. R. Kreitzman, C. D. P. Levy, R. Poutissou, R. H. Heffner, J. E. Elenewski, L. H. Greene, R. F. Kiefl. , Phys. Rev. Lett. 2004, 93, 157601.

[17] F. Hilmer, A. Schirmer, H. Ackermann, P. Heitjans, H.-J. Stöckmann, Z. Naturforsch. 1994, 49a, 271053. D please check, reference not found

[18] P. Heitjans, W. Faber, A. Schirmer, J. Non-Cryst. Solids 1991, 131-133, 1053.

[19] J. P. Hallett, T. Welton, Chem. Rev. 2011, 111, 3508.

[20] J. J. R. Frausto da Silva, R. J. P. Williams in The biological chemistry of the elements, Oxford University Press, 2001.

[21] J. C. C. Freitas, M. E. Smith, Annu. Rep. NMR Spectrosc. 2012, 75, 25.

[22] T. J. Bastow, S. Celotto, Solid State Nucl. Magn. Reson. 2009, 35, 217.

Received: September 5, 2014

Published online on $\square \mathbf{\square}, 2014$ 


\section{COMMUNICATIONS}

Powerful attraction: ${ }^{31} \mathrm{Mg} \beta$-NMR spectra are measured for as few as $10^{7}$ magnesium ions in ionic liquid (EMIM-AC) within minutes, as a prototypical test case, in an external field of $0.3 \mathrm{~T}$ (left). The experimental setup (right) includes an incoming nuclear-spin-polarized ${ }^{31} \mathrm{Mg}^{+}$ion beam, a sample (indicated as a drop), $\beta$-particle scintillation detectors, and an NMR magnet.

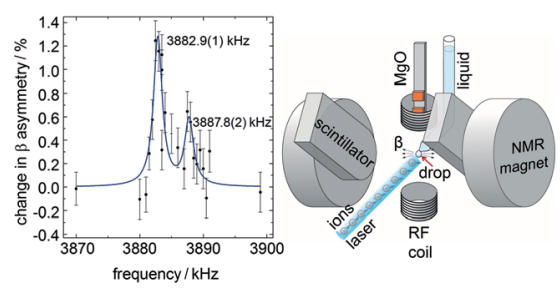

A. Gottberg, M. Stachura, * M. Kowalska, M. L. Bissell, V. Arcisauskaite, K. Blaum, A. Helmke, K. Johnston, K. Kreim, F. H. Larsen, R. Neugart, G. Neyens, R. F. G. Ruiz, D. Szunyogh, P. W. Thulstrup, D. T. Yordanov, L. Hemmingsen*

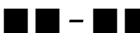

Billion-Fold Enhancement in Sensitivity of Nuclear Magnetic Resonance Spectroscopy for Magnesium Ions in Solution

Stachura/Hemmingsen \& co-workers achieve billion-fold increase in \#NMR sensitivity of \#Mg ions SPACE RESERVED FOR IMAGE AND LINK

ChemPhysChem is piloting a social networking feature involving Twitter, an online microblogging service that enables its users to send and read text-based messages of up to 140 characters, known as "tweets". Please check the pre-written tweet in the galley proofs for accuracy. Should you or your institute have a Twitter account, please let us know the appropriate username (e.g., @ChemPhysChem), and we will do our best to include this information in the tweet. This tweet will be posted to the journal's Twitter account upon online publication of your article.

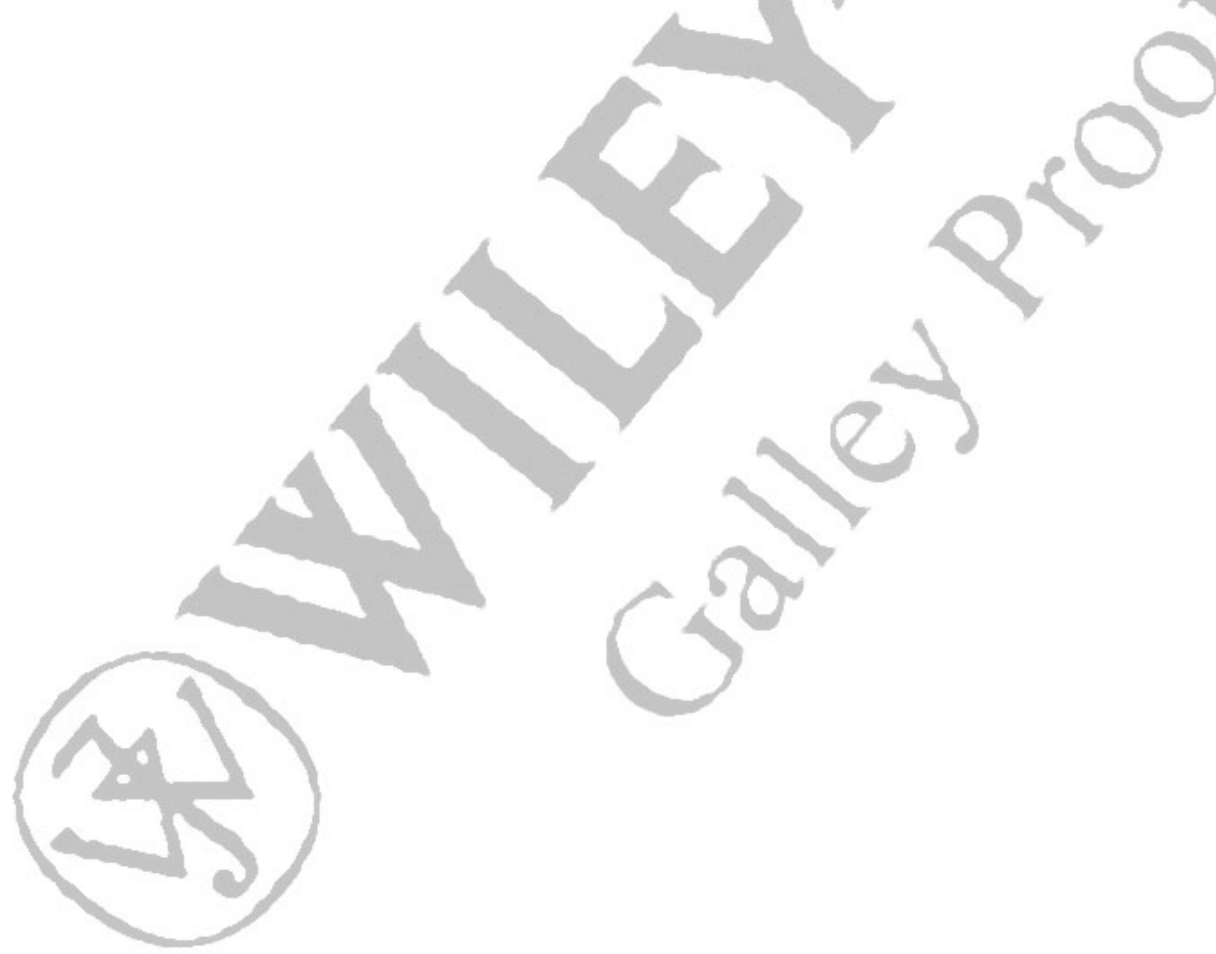

